\title{
1 Assembly and application of a low budget 2 photo reactor
}

3

4 Olov Wallner ${ }^{1, *}, \#$, Kirill Mamonov ${ }^{1, \#}$, Florian Ortis ${ }^{1, \#}$, Dana Michel ${ }^{2}$ and Maurice Michel ${ }^{1, *}$

$5{ }^{1}$ Science for Life Laboratory and Department of Oncology and Pathology, Karolinska Institutet, Solna, 6 Sweden

${ }^{2}$ Chemical Processes \& Pharmaceutical Development, Unit Process Chemistry I, Research Institutes of Sweden - RISE, 15136, Södertälje, Sweden

To whom correspondence should be addressed: olov.wallner@ki.se; maurice.michel@ki.se

\# contributed equally

\section{Abstract}

Photochemistry is a fast growing research field and many transformations previously not accessible to chemists now have become part of an ever growing standard repertoire. The limiting factors for a photo reactor system however is the possibility to perform stirring, removal of excess heat and the irradiation with UV or visible light - all that within a secure surrounding. Systems for starters may be as expensive as several thousand Euro. Here we design and assemble a LED photo reactor using scrap and standard materials, only spending less than $30 €$ for a LED. The system may be adjusted to any required wave length and its assembly is shown for the use of a $400 \mathrm{~nm}$ wave length lamp. To demonstrate its application, we then exemplarily use the reactor in the removal of a photo labile protection group during the synthesis of a SARS-CoV-2 spike protein glyco peptide.

Keywords: UV, photo reactor, photo labile, protection group, SARS-Cov-2, spike protein

\section{Introduction}

Photo reactors are broadly used in modern technology. Industrial use spans from waste water treatment ${ }^{1}$ to the production of food $^{2,3}$ and applications in material science, chemistry ${ }^{4}$ and biochemistry are steadily increasing. This lead to the development of new research fields around the use of for example UV light. Chemical reactions induced by UV/visible light are key to access a plethora of new reactions and modifications previously not accessible by chemists. Next to the development of novel catalysts the driving force for the use of UV/Vis photo reactors has been its application in flow chemistry. Here, advantages in energy and material transfer but also the altered kinetic characteristics 
of the system may be readily exploited. Consequently, research labs focusing entirely on the matter have developed, rapidly advancing photochemistry and flow chemistry as an intertwined research field. However, limiting factor for a laboratory to engage in UV light induced chemical reactions remains the access to a safely operable system. Commercial options are in the range of several thousand Euro and are often considered too costly to attempt singular chemical reactions. Here, we report on the design and the assembly of a low budget UV-Vis photo reactor. The system in its entirety was assembled from scrap material found at the recycling station, acquiring only a commercially available LED and a fan speed regulator for the total price of 30 Euro. To show its applicability we then used the system in the removal of a photo labile protection group in solid phase peptide synthesis.

\section{Design and Assembly}

\section{The frame}

The reactor was planned as a two compartment system within an old subwoofer box - one for electrics taking one third of available space and the other as a chemical environment with the remainder of space big enough to prevent temperature increase around the reaction vessel and to support an HPLC or standard test tube rack (Figure 1A). Within a suitably sized box, medium density fiberboard was used to separate rooms and allow quick modifications. Both compartments received a number of holes to ensure steady ventilation and removal of excess heat by the installed fan system (Figure $1 \mathrm{~A}$ and $1 B$ ). Another hole was carved into the connecting wall to allow placement of the LED supported by an active cooling system (Figure 1C and 1D). The front of the electrical compartment received a wall and two holes to support switches for main and LED (Figure 1E). Next, the chemistry compartment received a hinge operated door - the width was chosen to ensure passage of a standard HPLC or test tube rack, the height for their corresponding reaction vessels (Figure 1F). Further, an exhaust hole for a second CPU fan was carved into the bottom under the reaction space in front of the LED (Figure 1G). All holes in the fiber board were matched with corresponding holes within the old subwoofer box (Figure $1 \mathrm{H}$ ). With the supporting frame assembled, installation of the electric system could commence.

\section{Fans, Lamp and Stirring}

To enable heat transport away from the electric systems a passive heatsink with a cooling CPU fan was installed within the connection hole from the electrical to the chemical compartment (Figure 2A). The fan was anchored vertically into the floor of the fiber board. On the heatsink the LED was installed to irradiate the space over the exhaustion hole. There a CPU fan was installed to exhaust air and to allow stirring of reactions (Figure 2B). A sufficiently big stir bar was removed from its PTFE coating and glued to the center of the CPU fan. To complete work on the chemical compartment, a folded metal sheet 
was attached to the ground to enable reflection of scattered light, swift mounting of the reaction rack and to prevent the rack from hitting the LED (Figure 2C).

The wiring

The wiring was integrated as shown in Figure 4. A fuse was installed after the $230 \mathrm{~V}$ input, followed by a Double Pole Double Throw Switch (DPDT). The latter enabled the installation of two cycle set up. The first cycle consisted of another switch attached to the LED. The second cycle was wired to a $12 \mathrm{~V}$ DC transformer for voltage regulation. Within this cycle the LED fan and the fan bearing the magnetic stir bar were separated again. An installed fan speed regulator allowed for suitably chosen rounds per minutes applicable to stirring a chemical reaction.

\section{The assembled reactor and accessories}

Once the wiring was completed, the reactor was placed in the subwoofer box - note the alignment of fan and holes for ventilation and the switches together with hatch placed at the front (Figure 5A and 5B). When switched on, the light is reflected by the metal sheet to increase irradiation (Figure $5 \mathrm{C}$ and 5D). A HPLC rack mounted with a reaction vessel was marked for best irradiation for up to three different positions or reactions at once (Figure 5E and 5F). Additionally, a mirror was glued to the wall opposite the LED to reflect the light back towards the reaction vessels (not shown).

\section{Application in peptide synthesis}

In the synthesis of post translationally modified peptides, site selective removal of protection groups is often achieved by catalytic measures. This is necessary due to the properties of Fmoc-SPPS which requires basic conditions for peptide bond formation and acidic conditions for cleavage of amino acid protection groups. Structurally diverse post translational modifications beyond a single unit are therefore difficult to achieve in a one molecule approach and elegant alternatives have thus been developed in the past. ${ }^{5}$ During our efforts of performing glyco peptide enabled serology ${ }^{6}$ we aimed to synthesize diglycosylated peptides of the SARS-Cov-2 spike glycoprotein, especially those sequences close to the receptor binding domain. We chose the sequence FNATR (6X6P, aa342-346) ${ }^{7}$ as a model peptide to establish a previously reported photo labile protection group ${ }^{8}$ in the synthesis of sequences from the spike glycoprotein. Since the incorporation of glycans on already synthesized peptide backbones occurs via Lansbury coupling, the asparagine amino acid was replaced by aspartate. ${ }^{9,10}$ The presence of now two carboxylic acids was resolved by protecting one with the mentioned photo labile group, called 4-(Dimethyl)-aminophenacyl (Map, Scheme 1). After using standard amino acids, the photo labile protected building block and the Fmoc-Ala-Thr(psiMe,Mepro ${ }^{11}$ to synthesize the sequence FDATR on resin, a test cleavage was performed (Figure 6). With the peptide successfully 
synthesized, the photo labile group was removed by stirring resin corresponding to $0.05 \mathrm{mmol}$ in a 1:1 mixture of DCM and Ethanol and under irradiation in the photo reactor. After 16 hours, the solvent was removed and a test cleavage was performed. The observed full removal of the protection group and traces of the corresponding pseudo proline ${ }^{11}$ demonstrated the suitability of the low budget photo reactor for the removal of photo labile protection groups (Figure 7).

\section{Summary}

Here, we have shown the design and assembly of a LED based photo reactor for the wavelength of $400 \mathrm{~nm}$. Afterwards, we have successfully applied the reactor in the removal a photo labile protection group during solid phase peptide synthesis. Although the reactor outlined her is equipped to perform reactions at $400 \mathrm{~nm}$, its modular design allows for the adaptation to other wave lengths in the future. In summary, we have outlined a safe and inexpensive strategy for access to photo chemistry.

\section{Materials and Methods}

\section{Reactor}

Box (subwoofer, PC tower, etc.), CPU cooling fan, CPU heatsink, Medium density fiberboard, 12V transformer, CPU fan speed regulator, metal sheet, commercial LED (10W, $400 \pm 5 \mathrm{~nm}$ ), cables, electrical switches and connectors, screws, HPLC-tube rack, glue, chemistry equipment

\section{Synthetic Chemistry}

All reagents and solvents were purchased from Activate Scientific, Sigma-Aldrich, Bachem, Iris, CombiBlocks, Thermo Fischer Scientific or VWR and were used without purification. Analytical LC-MS were performed on an Agilent Technologies LC/MSD mass spectrometer connected to an Agilent 1260 Infinity II system with: Method A00100_15: Column ACE 3 C8 $(50 \times 3.0 \mathrm{~mm}) ; \mathrm{H}_{2} \mathrm{O}(+0.1 \%$ TFA) and MeCN were used as mobile phases at a flow rate of $1 \mathrm{ml} / \mathrm{min}$, with a gradient from $0 \%-100 \%$ in 15 min. For LC-MS, detection was made by UV (254 or 180-305 nm) and MS (ESI+).

\section{Photo labile building block synthesis}

Synthesis of the photo labile building block was performed as reported here ${ }^{8}$ (pages 143-145) with the exception of starting from commercially available 2-bromo-1-(4-(dimethylamino)phenyl)ethanone. The compound was stored in a cold place under exclusion of light.

\section{Synthesis of Peptide and Test cleavage}

Synthesis of Fmoc-FD(Map)ATR was performed in $0.1 \mathrm{mmol}$ scale on a Trityl-Resin in a syringe with double coupling of Standard amino acid building blocks (15 min each) and double coupling of 
Pseudoproline Fmoc-Ala-Thr(psiMe,Mepro) and Fmoc,Map-Asp-OH (30 min each). PYBOP and DIPEA were used for activation, DMF as solvent. The synthesized peptide was washed on resin with DCM and EtOH and dried under High-vacuum for storage in a dark cold place.

For Map cleavage, the resin was suspended in a 1:1 mixture of DCM and EtOH and the suspension was stirred for 16 hours under irradiation with $400 \mathrm{~nm}$ light.

For a test cleavage, the resin was suspended in TFA:TIPS: $\mathrm{H}_{2} \mathrm{O}$ (90:5:5) for $4 \mathrm{~h}$. The resulting solution was filtered and the filtrate was reduced to dryness in a nitrogen stream. The residue was dissolved in $\mathrm{ACN}: \mathrm{H}_{2} \mathrm{O}(50: 50)$ and injected into the LCMS.

\section{Contribution}

O.W. designed and assembled the photo reactor. K.M. and F.O. synthesized peptides and Fmoc-SPPS building blocks. D.M. and M.M. rationalized, planned and supervised synthesis and performed in-silico sequence assessment. M.M. wrote the manuscript. All authors commented on and approved the final version of the manuscript.

\section{Acknowledgement}

We would like to thank Ulrika Warpman Berglund and Thomas Helleday for their support and freedom to support critical research. Louise Sjöholm, Teresa Sandvall, Mari Kullman Magnusson, Kristina Edfeldt, Michael Sundström and Franz Ferdinand are acknowledged for their technical support. This work was funded by a Postdoctoral Stipend of the Thomas Helleday Foundation for medical research (M.M.), the EU/EFPIA/OICR/McGill/KTH/Diamond Innovative Medicines Initiative 2 Joint Undertaking (EUbOPEN grant $n^{\circ}$ 87551, M.M.), Lars Hiertas Minne Stiftelse (M.M) and Längmanska Kulturfonden (M.M).

\section{References}

1. Ramos, S., Homem, V., Alves, A. \& Santos, L. A review of organic UV-filters in wastewater treatment plants. Environment International 86, 24-44 (2016).

2. Delorme, M. M. et al. Ultraviolet radiation: An interesting technology to preserve quality and safety of milk and dairy foods. Trends in Food Science \& Technology 102, 146-154 (2020).

3. Hirneisen, K. A. et al. Viral Inactivation in Foods: A Review of Traditional and Novel FoodProcessing Technologies. Comprehensive Reviews in Food Science and Food Safety 9, 3-20 (2010). 
4. Cambié, D., Bottecchia, C., Straathof, N. J. W., Hessel, V. \& Noël, T. Applications of ContinuousFlow Photochemistry in Organic Synthesis, Material Science, and Water Treatment. Chem. Rev. 116, 10276-10341 (2016).

5. Mollica, A., Pinnen, F. \& Costante, S. A. and R. The Evolution of Peptide Synthesis: From Early Days to Small Molecular Machines. Current Bioactive Compounds vol. 9 184-202 https://www.eurekaselect.com/119784/article (2013).

6. Roxhed, N. et al. A translational multiplex serology approach to profile the prevalence of antiSARS-CoV-2 antibodies in home-sampled blood. medRxiv 2020.07.01.20143966 (2020) doi:10.1101/2020.07.01.20143966.

7. Herrera, N. G. et al. Characterization of the SARS-CoV-2 S Protein: Biophysical, Biochemical, Structural, and Antigenic Analysis. bioRxiv 2020.06.14.150607 (2020) doi:10.1101/2020.06.14.150607.

8. Michel, D. Synthesis of homogeneous N-glycosylated- and GPI-anchored peptides for semisynthesis of the prion protein. (2018).

9. Anisfeld, S. T. \& Lansbury, P. T. A convergent approach to the chemical synthesis of asparaginelinked glycopeptides. J. Org. Chem. 55, 5560-5562 (1990).

10. Cohen-Anisfeld, S. T. \& Lansbury, P. T. A practical, convergent method for glycopeptide synthesis. J. Am. Chem. Soc. 115, 10531-10537 (1993).

11. Heinlein, C. et al. Fragment Condensation of C-Terminal Pseudoproline Peptides without Racemization on the Solid Phase. Angewandte Chemie International Edition 50, 64066410 (2011). 


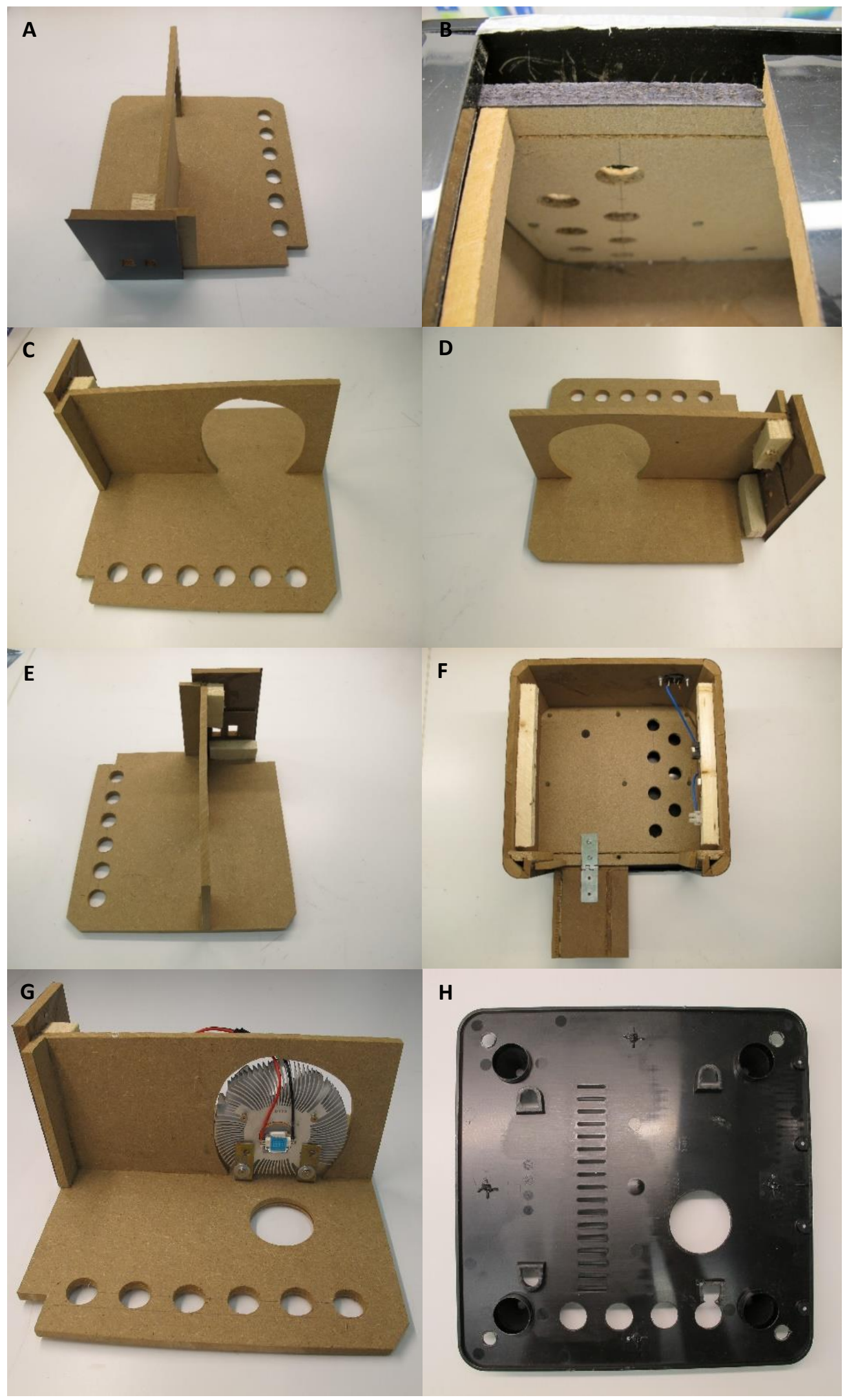

Figure 1: Implementation of the general frame of outer and inner box of the photo reactor. The interior is separated into a chemical and an electrical compartment. 


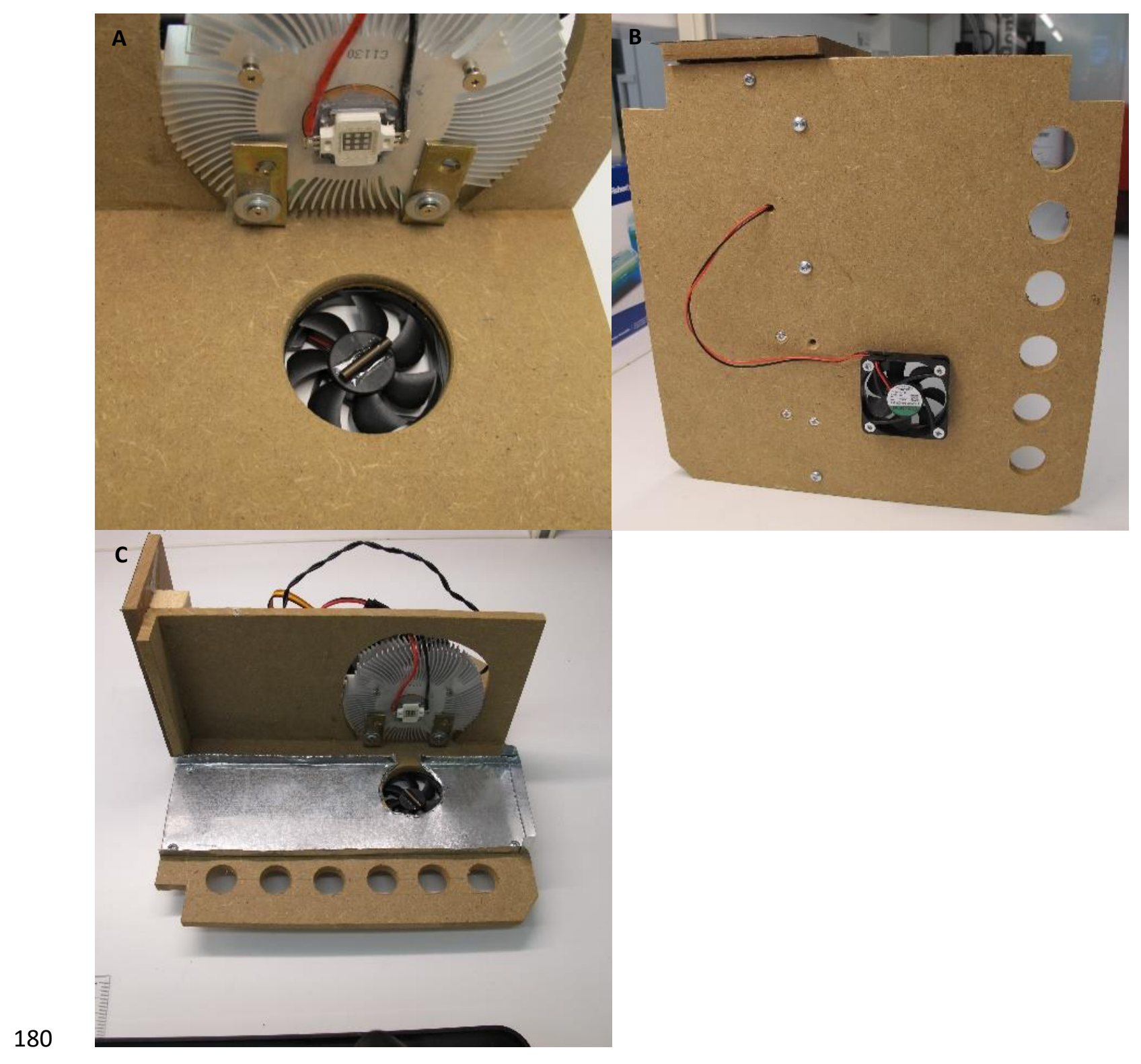

181 Figure 2: Installation of heatsink, Fans, stirring, UV-lamp and reflective shield. 


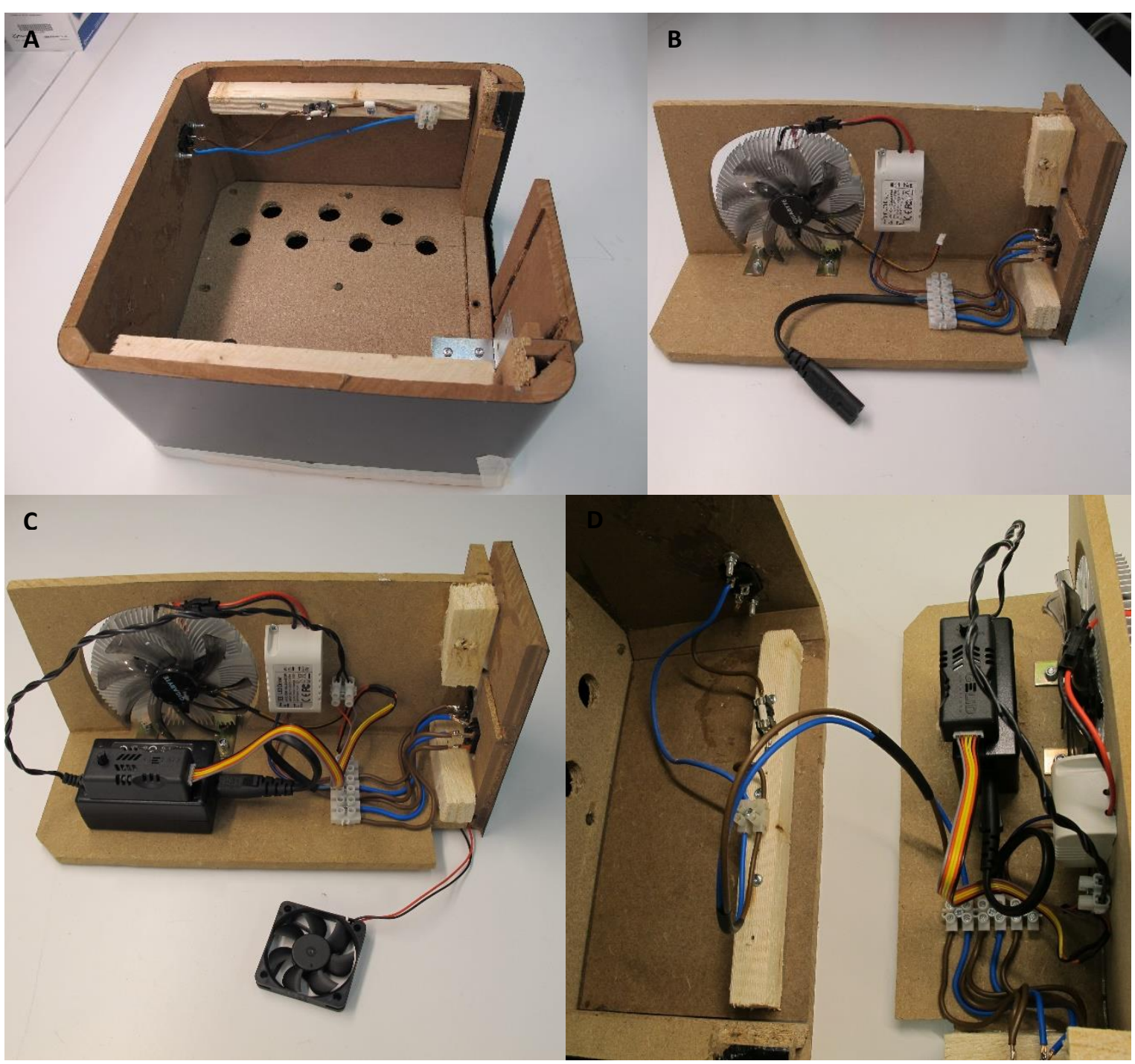

Figure 3: The wiring of the two compartments and their respective units is outlined in Figure 4. 


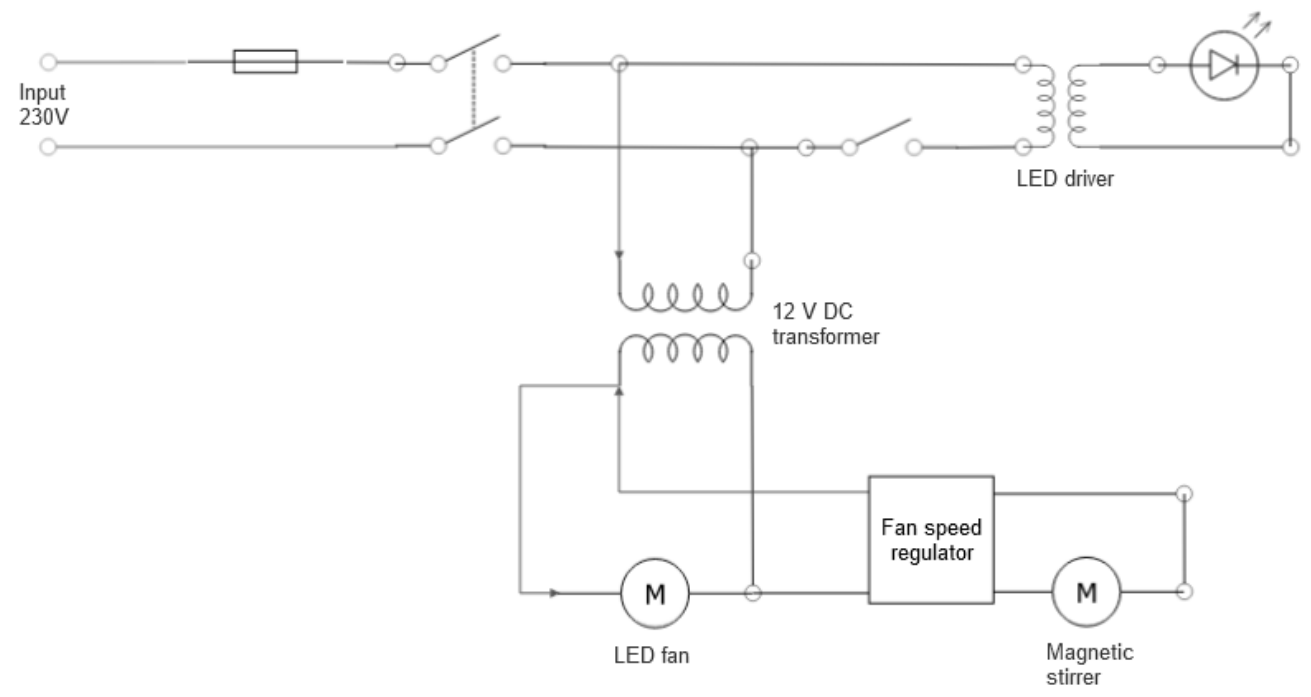


A

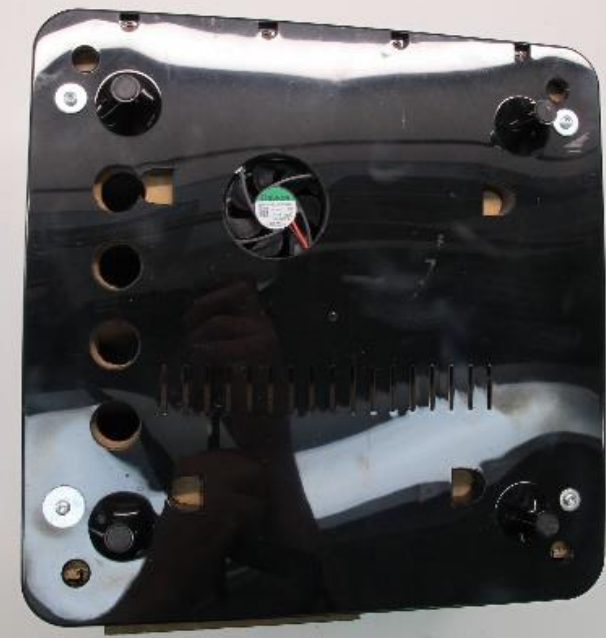

B
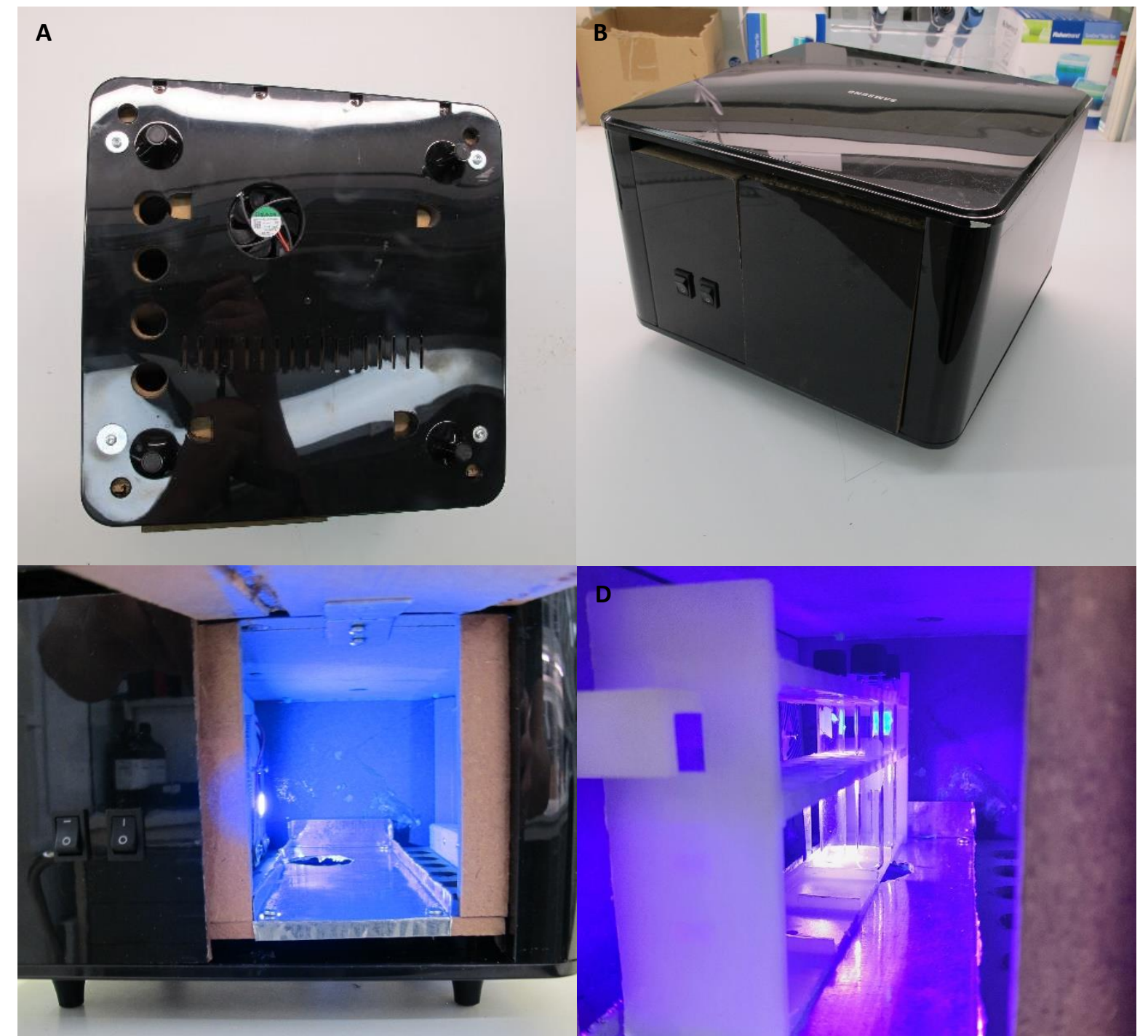

C
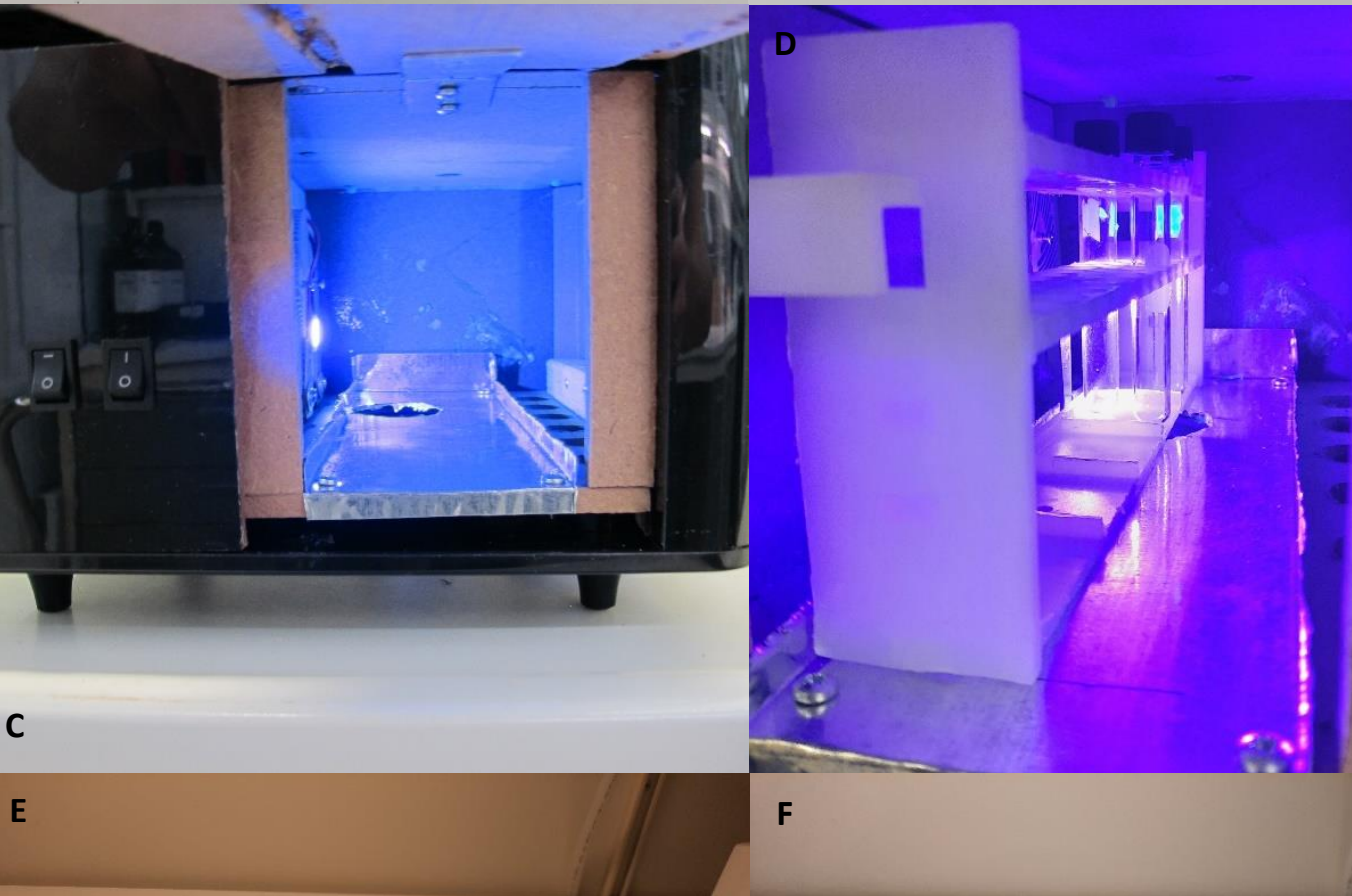

$\mathbf{F}$

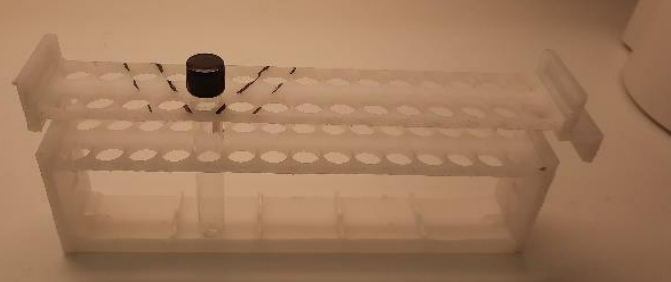

Figure 5: The assembled photo reactor and the reaction vessel. 


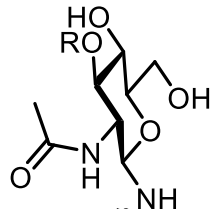

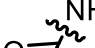

FNATR-
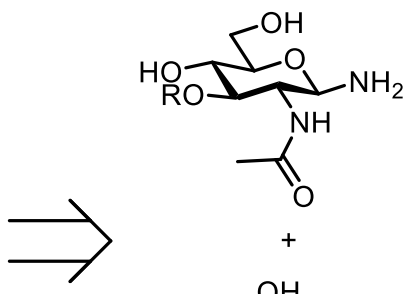

$\mathrm{O}=\mathrm{CH}^{\mathrm{H}}$

FDATR-

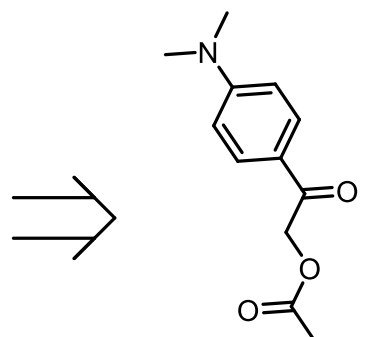

aа342-346
FDATR

189 Scheme 1: Possible retrosynthetic application of a photolabile protection group, 4-(Dimethyl)-aminophenacyl (Map).

190

191
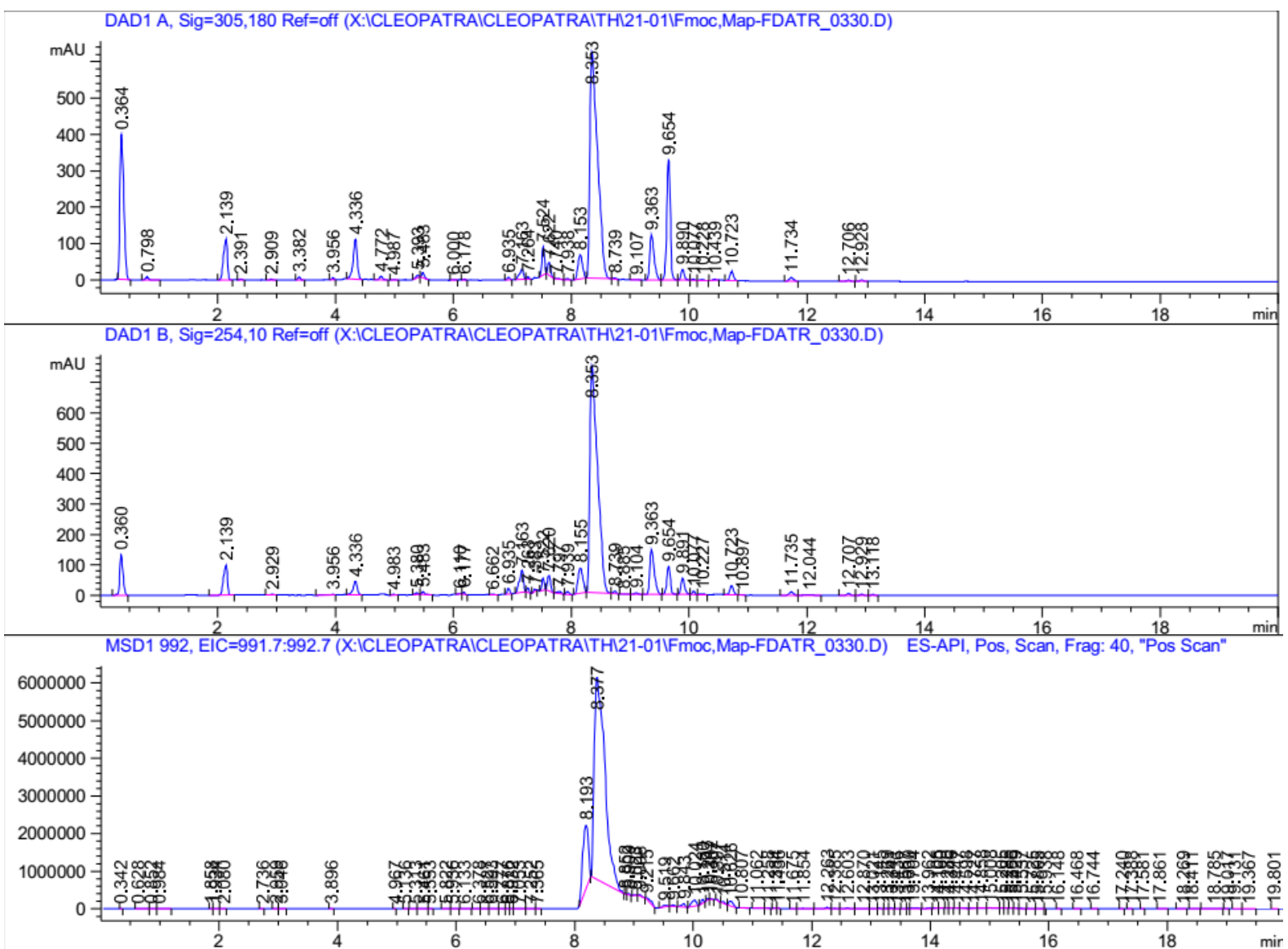

Figure 6: LCMS spectrum of a test cleavage of the peptide Fmoc-FD(Map)ATR ( $\mathrm{m} / \mathrm{z} 992$ ). 

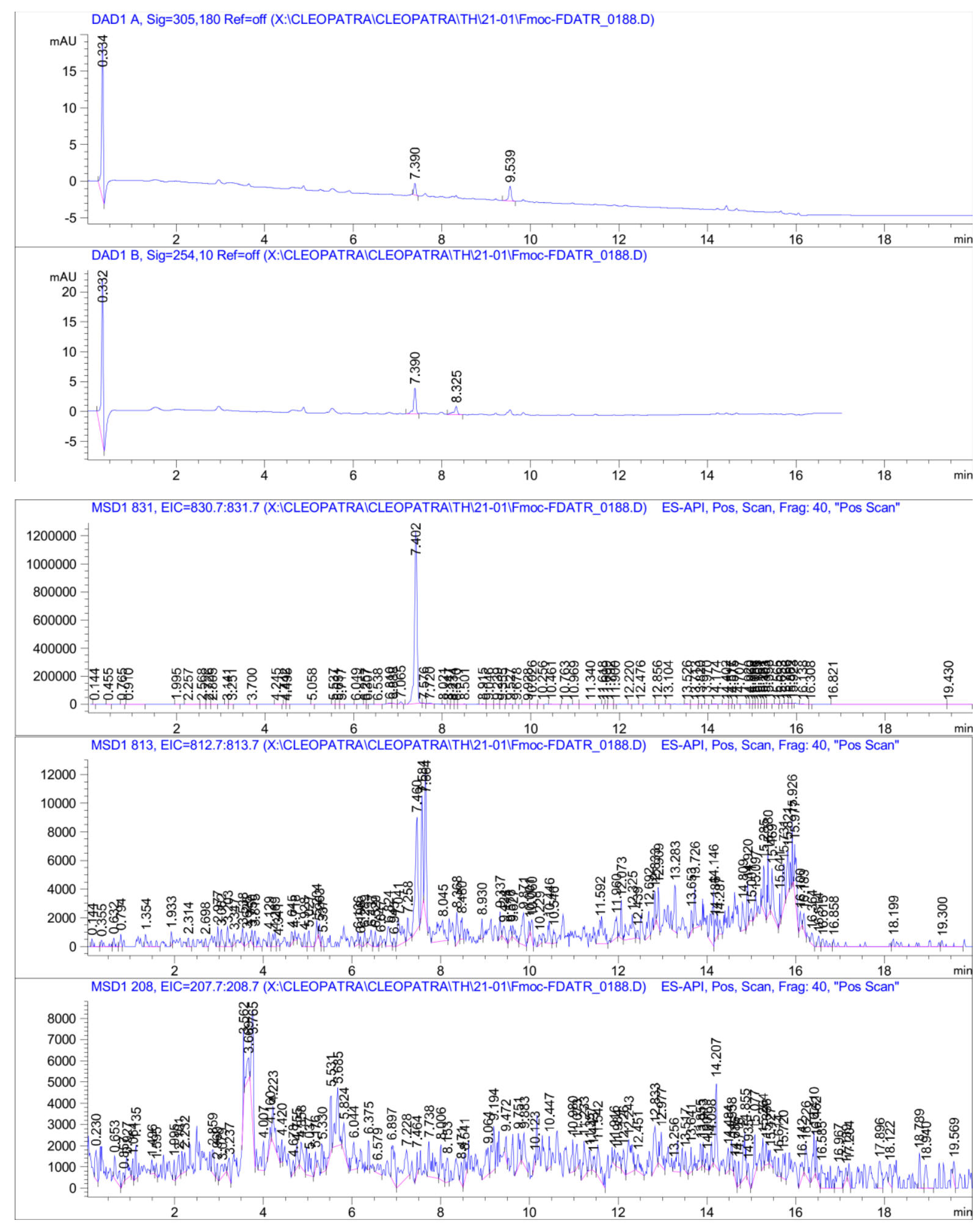

Figure 7: LCMS spectrum of a test cleavage of Fmoc-FDATR $(\mathrm{m} / \mathrm{z}=831)$, with $\mathrm{m} / \mathrm{z}=813$ highlighted for the aspartimid product. $\mathrm{m} / \mathrm{z}=208$ corresponds to the ethanol quenched photo labile protection group. 